\title{
Electromagnetic microwaves in metal films with electron-phonon interaction and a dc magnetic field
}

\author{
Hasselberg, L.E.
}

Published in:

Physical Review B

Link to article, DOI:

10.1103/PhysRevB.13.2324

Publication date:

1976

Document Version

Publisher's PDF, also known as Version of record

Link back to DTU Orbit

Citation (APA):

Hasselberg, L. E. (1976). Electromagnetic microwaves in metal films with electron-phonon interaction and a dc magnetic field. Physical Review B, 13(6), 2324-2330. https://doi.org/10.1103/PhysRevB.13.2324

\section{General rights}

Copyright and moral rights for the publications made accessible in the public portal are retained by the authors and/or other copyright owners and it is a condition of accessing publications that users recognise and abide by the legal requirements associated with these rights.

- Users may download and print one copy of any publication from the public portal for the purpose of private study or research.

- You may not further distribute the material or use it for any profit-making activity or commercial gain

- You may freely distribute the URL identifying the publication in the public portal

If you believe that this document breaches copyright please contact us providing details, and we will remove access to the work immediately and investigate your claim. 


\title{
Electromagnetic microwaves in metal films with electron-phonon interaction and a dc magnetic field
}

\author{
L. E. Hasselberg \\ Nordita, Blegdamsvej 17, DK-2100 Copenhagen 0, Denmark \\ and Physics Laboratory I, The Technical University of Denmark, DK-2800 Lyngby, Denmark
} (Received 9 September 1975)

\begin{abstract}
A quantum-mechanical treatment of electromagnetic microwaves is performed for a metal film. The directions of the exterior ac and dc fields are taken to be arbitrary and boundary conditions for the electrons are assumed to be specular. The relation between the current and the electromagnetic field becomes nonlocal both in ordinary and Fourier space. An expression for the electron self-energy is introduced, which includes electron-phonon interaction and an effective relaxation time. The theory is applied to cyclotron phase resonance in potassium. It is suggested that the experimental peak structure in the transmission spectrum can perhaps be obtained by assuming a finite Debye temperature and specular reflections of the electrons at the boundary surfaces. A sharp peak entirely caused by the finite electron-phonon interaction is also discussed.
\end{abstract}

\section{INTRODUCTION}

Most of the theoretical papers written about electromagnetic waves in metals with a dc magnetic field applied parallel to the metal surface (cyclotron resonance) or perpendicular to the surface (cyclotron phase resonance) have assumed a local relation between the Fourier transforms of the current and the electromagnetic field inside the metal (e.g., Antoniewitz ${ }^{1}$ ). However, if the metal has the geometry of a thin film with specularly reflecting boundary surfaces, Carolan and van Gelder ${ }^{2}$ and van Gelder ${ }^{3}$ have proposed that a nonlocal term obtained from linear quantum transport theory yields some contribution to the surface resistance. In this paper we will develop a somewhat more general theory including a finite self-energy and applicable to both cyclotron resonance and cyclotron phase resonance (CPR).

Fine structures in the dispersion relation, the transmission spectrum, and the surface impedance can be interpreted in different ways. One possibility is to introduce the semiphenomenological Fermi-liquid parameters (e.g., Platzman and $\mathrm{Jacobs}^{4}$ ) and another is to assume finite electronelectron interaction (e.g., Rice ${ }^{5}$ ) or electronphonon interaction (e.g., Prange and Kadanoff ${ }^{6}$ ). Anisotropies in the Fermi surface (e.g., Fredkin and Freedman ${ }^{7}$ ) also may have effects on the electromagnetic waves. In this paper we want to show that some elements of structure in the transmission spectrum are caused by the electron-phonon interaction. Others are related to the model including specular reflection of the electrons. We also want to compare with the CPR experiment by Phillips et al. ${ }^{8}$ They have found a sharp peak and some notches around it in the CPR transmission of potassium. By introducing Fermi-liquid parameters and neglecting the influence of the slab boundaries, they have suc- ceeded in obtaining a rather good fit with the experiments. Baraff ${ }^{9}$ has used a more sophisticated variational theory including diffuse boundaries and a couple of Fermi-liquid parameters. By the aid of a set of fitting parameters, he also has obtained a reasonable agreement with the CPR experiment.

Our aim is to obtain proper structures without introducing any model parameters, apart from the Debye temperature and the effective relaxation time. However, experimentally the re are two unknown parameters, namely the amplitude and the phase of the added bias signal which is incident on the detector (bolometer) by a separate path and which also includes the unavoidable leakage signal around the metal. The bias signal is assumed to be constant, when the magnetic field only is changing $10 \%$ around a mean value. Barafi $^{\kappa^{9}}$ does not take any account of the bias signal. Further, the amplitude and zero level of the total transmitted microwave power (transmission spectrum) are two other experimentally unknown quantities.

In Sec. II we derive a quantum-mechanical formalism for a metal film with specular boundary conditions. The exterior electromagnetic and dc magnetic fields are directed arbitrary relative to the film, in order to show that the same formalism can be used in the cyclotronresonance and CPR cases. The Fermi surface is assumed to be spherical and the theory can therefore be applied to potassium. The relation in the Fourier space between the current and the electric field becomes nonlocal. The self-energy is given its special form in Sec. III, where we also specialize to CPR waves. The expression for the electron-phonon interaction is constructed from the self-energy obtained by Scher and Holstein ${ }^{10}$ and contains only the Debye temperature and the effective relaxation time as parameters.

In Sec. IV the transmission coefficient (no bias 
signal) is calculated by iterating the nonlocal equation a couple of times until an associated sum rule is sufficiently satisfied. The numerical calculations are performed without any approximations apart from the fact that the number of iterations is finite. Different film thicknesses are tested, but the computer limits the thickness to about $1 \mu \mathrm{m}$, which is much smaller than the experimental one $(32.5 \mu \mathrm{m})$. Different efforts to perform approximate calculations with the experimental thickness have failed because of the slow decrease of the terms in the final Fourier sums. However, one fact is evident; namely, that the peak structure becomes more prominent for thicker films. A proper bias signal is added to the direct transmitted signal (transmission coefficient) and the total transmitted microwave power is plotted. The magnitude of the width of a very sharp transmission peak caused by the electron-phonon interaction is also estimated.

Finally, in Sec. V we discuss the structures obtained in Sec. IV for the different film thicknesses both with and without electron-phonon interaction. Further, we suggest that the sharp peak in the CPR experiment by Phillips et al. ${ }^{8}$ can perhaps be calculated by the aid of this microscopic theory and a large and very rapid computer.

\section{CURRENT EXPRESSION WITH ARBITRARY DIRECTIONS OF EXTERIOR FIELDS}

The geometry of the problem is the following: The metal has the boundaries $x=0, x=L_{x}, y=0$, $y=L_{y}, z=0, z=L_{z}$, where $L_{x}, L_{y} \gg L_{z}$. The $x$ axis is chosen perpendicular to the dc magnetic field

$$
\overrightarrow{\mathrm{B}}^{\mathrm{dc}}=B(0, \sin \alpha, \cos \alpha) \text {. }
$$

The corresponding vector potential is

$$
\overrightarrow{\mathrm{A}}^{\mathrm{dc}}(x)=B x(0, \cos \alpha,-\sin \alpha) \text {. }
$$

The general expression we will use for the current density of "spin up" electrons is

$$
\begin{aligned}
\overrightarrow{\mathrm{j}}_{\mathfrak{r}}(\overrightarrow{\mathrm{r}}, t)= & \left\langle\overrightarrow{\mathrm{j}}_{i}^{\mathrm{op}}(\overrightarrow{\mathrm{r}}, t)\right\rangle=\frac{-e}{2 m} \int d^{3} r^{\prime}\left\langle\Psi^{\dagger}\left(\overrightarrow{\mathrm{r}}^{\prime}, t\right)\right. \\
& \left.\times\left\{\delta\left(\overrightarrow{\mathrm{r}}-\overrightarrow{\mathrm{r}}^{\prime}\right),-i \hbar \frac{\partial}{\partial \overrightarrow{\mathrm{r}}^{\prime}}+e \overrightarrow{\mathrm{A}}\left(\overrightarrow{\mathrm{r}}^{\prime}, t\right)\right\} \Psi\left(\overrightarrow{\mathrm{r}}^{\prime}, t\right)\right\rangle,
\end{aligned}
$$

where $\vec{A}(\vec{r}, t)$ is the total time-dependent vector potential and the superscript op stands for "operator." $\langle\cdots\rangle$ means an average in the grand canonical ensemble and the curly brackets indicate an anticommutator. The field operators $\Psi(\vec{r}, t)$ are expanded in the Landau representation of the single-particle states

$$
\Psi(\overrightarrow{\mathrm{r}}, t)=\sum_{s} \Phi_{s}(\overrightarrow{\mathrm{r}}) a_{s}(t)
$$

where $a_{S}(t)$ are annihilation operators and $\Phi_{S}(\overrightarrow{\mathbf{r}})$ are eigenfunctions to the Hamiltonian $[-i \hbar(\partial / \partial \overrightarrow{\mathbf{r}})$ $\left.+e \overrightarrow{\mathrm{A}}^{\mathrm{dc}}(x)\right]^{2} / 2 m$ with the eigenvalues

$$
\begin{aligned}
\epsilon_{S}= & \hbar \omega_{c}\left(n+\frac{1}{2}\right)+\left(\hbar^{2} / 2 m\right)\left[k_{y}^{2}+k_{z}^{2}\right. \\
& \left.-\left(k_{y} \cos \alpha-k_{z} \sin \alpha\right)^{2}\right],
\end{aligned}
$$

and the quantum numbers

$$
\begin{aligned}
& S=\left[n, k_{y}, k_{z}\right] \\
& \left(n=0,1,2, \ldots ; m \omega_{c}=e B ; e>0\right) .
\end{aligned}
$$

The boundary conditions in the $x$ and $y$ directions are supposed to be periodic and in the $z$ direction specular. The explicit expressions for the normalized eigenfunctions are

$$
\begin{aligned}
\Phi_{S}(\overrightarrow{\mathrm{r}})= & \left(L L_{y} L_{z}\right)^{-1 / 2} \varphi_{n}\left(x / L+L k_{y} \cos \alpha\right. \\
& \left.-L k_{z} \sin \alpha\right) e^{i k_{y} y} \sin k_{z} z,
\end{aligned}
$$

with

$$
\begin{aligned}
& \left(k_{y}, k_{z}\right)=\left(2 \pi / L_{y}, \pi / L_{z}\right) \times \text { integer, } \\
& L=(\hbar / e B)^{1 / 2},
\end{aligned}
$$

and

$$
\varphi_{n}(x)=\left(\sqrt{\pi} 2^{n} n !\right)^{-1 / 2} e^{-x^{2} / 2} H_{n}(x)
$$

( $H_{n}$ are Hermite polynomials).

Now we superpose a weak electromagnetic field with the vector potential $\overrightarrow{\mathrm{A}}^{\mathrm{ac}}(\overrightarrow{\mathrm{r}}, t)$ and the corresponding electric field

$$
\overrightarrow{\mathrm{E}}(\overrightarrow{\mathrm{r}}, t)=-\frac{\partial}{\partial t} \overrightarrow{\mathrm{A}}^{\mathrm{ac}}(\overrightarrow{\mathrm{r}}, t) .
$$

The linear response of $\vec{j}_{t}$ to $\vec{A}^{\text {ac }}$ has a diamagnetic term, where $\Psi$ is unperturbed and $\vec{A}$ is perturbed in Eq. (2.3):

$$
\overrightarrow{\mathrm{j}}_{\mathrm{i}}^{\mathrm{dia}}(\overrightarrow{\mathrm{r}}, t)=\frac{i e}{m} \sum_{S}\left|\Phi_{S}(\overrightarrow{\mathrm{r}})\right|^{2} G_{S}^{<}(0) e \overrightarrow{\mathrm{A}}^{\mathrm{ac}}(\overrightarrow{\mathrm{r}}, t),
$$

where for negative values of $t, G_{S}^{<}(t)$ is identical to

$$
G_{S}(t)=-i\left\langle\tilde{T} a_{S}(t) a_{S}^{\dagger}(0)\right\rangle
$$

and $\tilde{T}$ is a time-ordering operator. The total Hamiltonian for the spin up electrons becomes augmented by

$$
H_{\mathrm{e} \cdot \mathrm{m} \cdot}(t)=-\int d^{3} r \overrightarrow{\mathrm{j}}_{i}^{\mathrm{op}}(\overrightarrow{\mathrm{r}}, t) \overrightarrow{\mathrm{A}}^{\mathrm{ac}}(\overrightarrow{\mathrm{r}}, t) .
$$

A first-order perturbation theory according to Keldysh ${ }^{11}$ yields the paramagnetic term of the current

$$
\begin{aligned}
\overrightarrow{\mathrm{j}}_{\mathrm{r}}^{\mathrm{para}}(\overrightarrow{\mathrm{r}}, t)= & \frac{i}{\hbar} \sum_{s_{1} s_{2}} \overrightarrow{\mathrm{b}}_{s_{1} s_{2}}(\overrightarrow{\mathrm{r}}) \int d^{3} r^{\prime} \overrightarrow{\mathrm{b}}_{s_{2} s_{1}}\left(\overrightarrow{\mathrm{r}}^{\prime}\right) \\
& \cdot \int_{-\infty}^{\infty} d t^{\prime} \overrightarrow{\mathrm{A}}^{\mathrm{ac}}\left(\overrightarrow{\mathrm{r}}^{\prime}, t^{\prime}\right)
\end{aligned}
$$




$$
\begin{aligned}
\times\left[G_{S_{2}}^{R}(t\right. & \left.-t^{\prime}\right) G_{S_{1}}^{<}\left(t^{\prime}-t\right) \\
& \left.+G_{S_{2}}^{<}\left(t-t^{\prime}\right) G_{S_{1}}^{A}\left(t^{\prime}-t\right)\right],
\end{aligned}
$$

where

$$
\begin{aligned}
b_{s_{1} s_{2}}(\overrightarrow{\mathrm{r}})=\frac{-e}{2 m} \int d^{3} r^{\prime} \Phi_{s_{1}}^{*}\left(\overrightarrow{\mathrm{r}}^{\prime}\right)\left\{\delta\left(\overrightarrow{\mathrm{r}}-\overrightarrow{\mathrm{r}}^{\prime}\right),-i \hbar \frac{\partial}{\partial \overrightarrow{\mathrm{r}}^{\prime}}\right. \\
\left.+e \overrightarrow{\mathrm{A}}^{\mathrm{dc}}\left(\overrightarrow{\mathrm{r}}^{\prime}\right)\right\} \Phi_{s_{2}}\left(\overrightarrow{\mathrm{r}}^{\prime}\right)
\end{aligned}
$$

The Green's functions have the Fourier transforms

$$
\begin{aligned}
\tilde{G}_{S}^{A}(E) & =\int_{-\infty}^{\infty} d\left(\frac{t}{\hbar}\right) e^{i E t / \hbar} G_{S}^{A}(t) \\
& =\left[E-\epsilon_{S}-\Sigma_{S}\left(E-i 0^{+}\right)\right]^{-1}, \\
\tilde{G}_{S}^{R}(E) & =\left[E-\epsilon_{S}-\Sigma_{S}\left(E+i 0^{+}\right)\right]^{-1}, \\
\tilde{G}_{S}^{<}(E) & =-f(E)\left[\tilde{G}_{S}^{R}(E)-\tilde{G}_{S}^{A}(E)\right] .
\end{aligned}
$$
and

$\Sigma_{S}$ is the self-energy caused by different kinds of interactions (for example electron-phonon interaction) and $f(E)$ is the Fermi distribution function

$$
f(E)=\left[\exp (E-\mu) / k_{B} T+1\right]^{-1},
$$

where $k_{B} T$ is Boltzmann's constant times the temperature, and $\mu$ is the chemical potential.

The spatial variation of $\overrightarrow{\mathrm{J}}, \overrightarrow{\mathrm{E}}$, and $\overrightarrow{\mathrm{A}}$ is limited to the $z$ direction because $L_{x}, L_{y} \gg L_{z}$. Therefore $\overrightarrow{\mathrm{b}}_{s_{1}, s_{2}}(\overrightarrow{\mathrm{r}})$ can be replaced by

$$
\begin{aligned}
\overrightarrow{\mathrm{b}}_{s_{1} s_{2}}(z) & =\int_{0}^{L_{x}} \int_{0}^{L_{y}} \frac{d x d y}{L_{x} L_{y}} \overrightarrow{\mathrm{b}}_{s_{1} s_{2}}(\overrightarrow{\mathrm{r}}) \\
& =\frac{-e \hbar}{m \Omega L} \delta_{k_{y_{1}}, k_{y_{2}}} \sin k_{z_{1}} z \sin k_{z_{2}} z \overrightarrow{\mathrm{V}}_{s_{1} s_{2}},
\end{aligned}
$$

where $\Omega$ is the volume. For large magnetic fields we have

$$
\overrightarrow{\mathrm{V}}_{s_{1} S_{2}} \approx \int_{0}^{L_{x} / L} d S\left(\begin{array}{c}
-\frac{1}{2} i\left(\varphi_{n_{1}} \varphi_{n_{2}}^{\prime}-\varphi_{n_{1}}^{\prime} \varphi_{n_{2}}\right) \\
\left(L k_{y_{1}}+S \cos \alpha\right) \varphi_{n_{1}} \varphi_{n_{2}} \\
-S \sin \alpha \varphi_{n_{1}} \varphi_{n_{2}}
\end{array}\right)
$$

$\left[\varphi_{n_{i}} \equiv \varphi_{n_{i}}\left(S+L k_{y_{i}} \cos \alpha-L k_{z_{i}} \sin \alpha\right) ; i=1,2\right]$.

A $z$-dependent term $\frac{1}{2} i L\left(k_{z_{1}} \cot k_{z_{1}} z-k_{z_{2}} \cot k_{z_{2}} z\right)$ $\times \varphi_{n_{1}} \varphi_{n_{2}}$ has been omitted in the $z$ component of $\overrightarrow{\mathrm{V}}_{S_{1}, s_{2}}$ for the following reason: If $\alpha$ is nonzero, this term yields a much smaller contribution than $-S \sin \alpha \varphi_{n_{1}} \varphi_{n_{2}}$ and if $\alpha$ is zero (the CPR case) the $z$ component of $\vec{V}_{S_{1}}, s_{2}$ is uninteresting because $\vec{j}$ and $\vec{E}$ are then transverse.

Because of the special $z$ dependence of $\vec{b}_{s_{1} s_{2}}(z)$ we introduce the cosine transform for the total current inclusive $\operatorname{spin}\left[q=\left(\pi / L_{z}\right) \times\right.$ integer $]$

$$
\begin{aligned}
& \vec{\jmath}(q, t)=\int_{0}^{L} z d z \cos q z\left[2 \overrightarrow{\mathrm{j}}_{\mathrm{i}}^{\mathrm{di} \mathrm{a}}(z, t)\right. \\
& \left.+2 \vec{j} \stackrel{p a r a}{i}^{p}(z, t)\right] \text {. }
\end{aligned}
$$

Further we assume that the time dependence of the electromagnetic field varies as $e^{-i \omega t}$. Then the current amplitude becomes $\vec{\jmath}(q)$ :

$$
\begin{aligned}
\vec{J}(q)= & \frac{e^{2}}{8 \pi \omega m \Omega} \sum_{s_{1} s_{2}} \delta_{k_{y_{1}}, k_{y_{2}}}\left(\delta_{q, k_{z_{1}}-k_{z_{2}}}-\delta_{q, k_{z_{1}}+k_{z_{2}}}\right) \overrightarrow{\mathrm{F}}_{s_{1} s_{2}} \\
& \times\left[\overrightarrow{\mathcal{E}}\left(k_{z_{1}}-k_{z_{2}}\right)-\overrightarrow{\mathcal{E}}\left(k_{z_{1}}+k_{z_{2}}\right)\right]+\{q \rightarrow-q\},
\end{aligned}
$$

where

$$
\begin{array}{r}
\overrightarrow{\mathrm{F}}_{s_{1} s_{2}}=\int_{-\infty}^{\infty} d E\left(\hbar \omega _ { c } \vec { \mathrm { V } } _ { s _ { 1 } s _ { 2 } } \vec { \mathrm { V } } _ { S _ { 2 } s _ { 1 } } \left[\tilde{G}_{S_{2}}^{R}(E+\hbar \omega) \tilde{G}_{S_{1}}^{<}(E)\right.\right. \\
\left.+\tilde{G}_{S_{2}}^{<}(E+\hbar \omega) \tilde{G}_{S_{1}}^{A}(E)\right]+\delta_{n_{1}, n_{2}} \tilde{G}_{S_{1}}^{<}(E) \\
\left.\times \int_{0}^{L_{x} / L} d S \varphi_{n_{1}}^{2} \overrightarrow{1}\right) .
\end{array}
$$

III. CPR WAVES WITH ELECTRON-PHONON INTERACTION

The self-energy is an analytic function and can therefore be written in the form

$$
\Sigma_{S}(Z)=\int_{-\infty}^{\infty} \frac{d \omega}{2 \pi} \frac{\Gamma_{S}(\omega)}{Z-\omega}
$$

with an infinitesimal imaginary part of $Z(Z$ $\left.=E \pm i 0^{+}\right)$:

$$
\Sigma_{S}\left(E \pm i 0^{+}\right)=M_{S}(E) \mp \frac{1}{2} i \Gamma_{S}(E),
$$

where

$$
M_{S}(E)=P \int_{-\infty}^{\infty} \frac{d \omega}{2 \pi} \frac{\Gamma_{S}(\omega)}{E-\omega}
$$

is the real part of the self-energy. According to Scher and Holstein ${ }^{10} \Gamma_{S}(E)$ varies much faster with the energy than with the quantum numbers and takes the approximate form

$$
\Gamma(E)=C\left[|W|^{3} \Theta(1-|W|)+\theta(|W|-1)\right],
$$

where $W=(\mu-E) / k_{B} \Theta_{D}, \Theta$ is the ordinary step function, and $\Theta_{D}$ is the Debye temperature. $C$ is the product of different constants and is given the value $2 \hbar / \tau$, where $\tau$ is the effective relaxation time for the electrons with energies fulfilling $|\mu-E|>k_{B} \Theta_{D}$. From Eq. (3.3) we get the corresponding expression

$$
M(E)=\frac{C}{2 \pi}\left(W+W^{3} \ln \left|\frac{1-W^{2}}{W^{2}}\right|+\ln \left|\frac{1+W}{1-W}\right|\right) .
$$

The relaxation time for energies very close to $\mu$ becomes very large. These electrons yield an enlarged transmission coefficient especially when $\omega_{c}$ is close to $\omega$. If $\omega \tau \gg 1$, the self-energy be- 
comes very small and $\tilde{G}_{S}^{<}$can be approximated by

$$
\tilde{G}_{S}^{<}(E) \approx 2 \pi i f(E) \delta\left(E-\epsilon_{S}\right) .
$$

Then $\overrightarrow{\mathrm{F}}_{s_{1}}, s_{2}$ can be rewritten [Eq. (2.24)]

$$
\begin{aligned}
\overrightarrow{\mathrm{F}}_{S_{1} s_{2}}= & 2 \pi i \int_{0}^{\infty} d E f(E)\left(\hbar \omega_{c} \overrightarrow{\mathrm{V}}_{s_{1} s_{2}} \overrightarrow{\mathrm{V}}_{S_{2} s_{1}}\right. \\
& \times \frac{\delta\left(E-\epsilon s_{1}\right)-\delta\left(E-\epsilon_{s_{2}}\right)}{\epsilon_{s_{1}}-\epsilon_{S_{2}}+\hbar \omega+\frac{1}{2} i \Gamma(E)} \\
& \left.+\delta\left(E-\epsilon_{s_{1}}\right) \delta_{n_{1}, n_{2}} \int_{0}^{L_{x} / L} d S \varphi_{n_{1}}^{2} \overrightarrow{1}\right),
\end{aligned}
$$

where we used the fact that

$$
\hbar \omega \ll \mu \text {. }
$$

Because of the rapid decrease of the function $\varphi_{n}$ in Eq. (2.21) for large arguments, the integration over $S$ from 0 to $L_{x} / L$ will yield an ap- proximate limit to the quantum numbers $k_{y}$ and $k_{z}$ :

$$
0<L k_{z} \sin \alpha-L k_{y} \cos \alpha<L_{x} / L \text {. }
$$

However, the inequalities $L_{x} \gg L$ and Eq. (3.9) make it possible to extend the integration from $-\infty$ to $+\infty$. Using four properties of $\varphi_{n}$, namely

$$
\begin{aligned}
& \varphi_{n}(x)=\frac{i^{-n}}{\sqrt{2 \pi}} \int_{-\infty}^{\infty} d y e^{i x y} \varphi_{n}(y), \\
& x \sqrt{2} \varphi_{n}(x)=\sqrt{n} \varphi_{n-1}(x)+\sqrt{n+1} \varphi_{n+1}(x), \\
& \sqrt{2} \frac{d}{d x} \varphi_{n}(x)=\sqrt{n} \varphi_{n-1}(x)-\sqrt{n+1} \varphi_{n+1}(x),
\end{aligned}
$$

$\int_{-\infty}^{\infty} d y e^{-i x y} \varphi_{n_{1}}(y) \varphi_{n_{2}}(y) \approx i^{n_{2}-n_{1}} J_{n_{1}-n_{2}}\left(x \sqrt{n_{1}+n_{2}}\right)$

$\left(n_{1}, n_{2} \gg 1 ; J_{n}\right.$ are Bessel functions), we can rewrite $\overrightarrow{\mathrm{V}}_{s_{1} s_{2}}$ in the following way:

$$
\overrightarrow{\mathrm{V}}_{S_{1} s_{2}}=\left(\begin{array}{c}
-\frac{1}{2} i \sqrt{n_{+}}\left(J_{n_{-}+1}-J_{n_{-}-1}\right) \\
\left(k_{y_{2}} \sin ^{2} \alpha+\frac{1}{2} k_{z_{2}} \sin 2 \alpha\right) L J_{n_{-}}+\frac{1}{2} \sqrt{n_{+}} \cos \alpha\left(J_{n_{-}+1}+J_{n_{-}-1}\right) \\
-\sin \alpha\left[\left(k_{z_{2}} \sin \alpha-k_{y_{2}} \cos \alpha\right) L J_{n_{-}}+\frac{1}{2} \sqrt{n_{+}}\left(J_{n_{-}+1}+J_{n_{-}-1}\right)\right]
\end{array}\right),
$$

where the argument of the Bessel functions is $L\left(k_{z_{2}}-k_{z_{1}}\right) \sin \alpha$ and

$$
n_{ \pm}=n_{1} \pm n_{2} \text {. }
$$

In order to obtain the CPR case we put $\alpha$ equal to zero. The problem becomes two-dimensional because the $z$ components of $\vec{j}$ and $\vec{E}$ are zero. The vector $\overrightarrow{\mathrm{V}}_{s_{1}, s_{2}}$ is reduced to

$$
\overrightarrow{\mathrm{V}}_{s_{1} s_{2}=\frac{1}{2} \sqrt{n_{+}}}\left(\begin{array}{c}
-i\left(\delta_{n_{-,-1}}-\delta_{n_{-, 1}}\right) \\
\delta_{n_{-},-1}+\delta_{n_{-}, 1}
\end{array}\right),
$$

and the product $\overrightarrow{\mathrm{V}}_{S_{1}, s_{2}} \overrightarrow{\mathrm{V}}_{s_{2}, s_{1}}$ in Eq. (3.7) can be diagonalized:

$\left(\begin{array}{cc}1 & i \\ 1 & -i\end{array}\right) \overrightarrow{\mathrm{V}}_{s_{1}, s_{2}} \overrightarrow{\mathrm{V}}_{s_{2} s_{1}}\left(\begin{array}{rr}1 & i \\ 1 & -i\end{array}\right)^{-1}=\frac{1}{2} n_{+}\left(\begin{array}{cc}\delta_{n_{-}, 1} & 0 \\ 0 & \delta_{n_{-},-1}\end{array}\right)$.

Therefore we can give the CPR case a one-dimensional form by introducing the amplitudes of the circularly polarized current and electric field

$$
j_{ \pm}=j_{x} \pm i j_{y} ; \quad E_{ \pm}=E_{x} \pm i E_{y} .
$$

At zero temperature and by the aid of Eq. (3.9), we can perform the summations over $n_{1}, n_{2}, k_{y_{1}}$, and $k_{y_{2}}$ :

$2 \mu H_{k_{z_{1}} k_{z_{2}}}^{ \pm} \equiv \frac{\hbar \omega L^{2}}{i L_{x} L_{y}} \sum_{\substack{n_{1} n_{2} \\ k_{y_{1} k_{y_{2}}}}} F_{S_{1} s_{2}}^{ \pm}$

$$
=\int_{0}^{\mu} \frac{d E\left[\hbar \omega+\frac{1}{2} i \Gamma(E)\right]}{\hbar \omega+\frac{1}{2} i \Gamma(E) \pm \hbar \omega_{c}+\left(\hbar^{2} / 2 m\right)\left(k_{z_{1}}^{2}-k_{z_{2}}^{2}\right)},
$$

and the final expression for the CPR current becomes

$$
\begin{aligned}
\jmath_{ \pm}(q)= & \frac{i Q^{2}}{2 \mu_{0} \omega} \sum_{k_{z_{1}} k_{z_{2}}} H_{k_{z_{1}} k_{z_{2}}}^{ \pm}\left(\delta_{q, k_{z_{1}}-k_{z_{2}}}+\delta_{q, k_{z_{2}}}{ }^{-k} z_{z_{1}}\right) \\
& \times\left[\mathcal{E}_{ \pm}\left(k_{z_{1}}-k_{z_{2}}\right)-\tilde{\mathcal{E}}_{ \pm}\left(k_{z_{1}}+k_{z_{2}}\right)\right],
\end{aligned}
$$

where

$$
Q^{2}=\left(\mu_{0} e^{2} / \pi \hbar^{2}\right) \mu / L_{\boldsymbol{z}} .
$$

$Q^{-1}$ has the dimension of length, and the magnitudes of $k_{z_{1}}$ and $k_{z_{2}}$ are limited by

$$
k_{F}=\sqrt{2 m \mu} / \hbar \text {. }
$$

\section{TRANSMISSION COEFFICIENT}

Maxwell's equations for the current and the field amplitudes yield

$$
\left[\frac{d^{2}}{d z^{2}}+\left(\frac{\omega}{c}\right)^{2}\right] E_{ \pm}(z)=-i \omega \mu_{0} j_{ \pm}(z),
$$

where the subscript \pm is omitted in the following. By the aid of partial integrations we obtain

$\left[q^{2}-(\omega / c)^{2}\right] \mathcal{E}(q)-i \omega \mu_{0} \mathcal{J}(q)=\cos q L_{z} E^{\prime}\left(L_{z}\right)-E^{\prime}(0)$. 
With an incoming wave of unit amplitude at $z=0$ and only outgoing waves at $z=L_{z}$, the boundary conditions are

$$
\begin{aligned}
& E(0)-i(c / \omega) E^{\prime}(0)=2, \\
& E\left(L_{z}\right)+i(c / \omega) E^{\prime}\left(L_{z}\right)=0,
\end{aligned}
$$

where

$$
E(z)=\frac{1}{L_{z}} \sum_{q} \cos q z \mathcal{E}(q) .
$$

Because of the right-hand side of Eq. (4.2), it is necessary to find one solution with even values of the integer $q L_{z} / \pi$ and one with odd values. The transmission coefficient $f$ is defined as

$$
f=E\left(L_{z}\right),
$$

and can be expressed as

$$
f=\left(P_{o}-P_{e}\right) /\left(1-P_{o}\right)\left(1-P_{e}\right),
$$

where

$$
\begin{aligned}
& P_{o(e)}=\sum_{q L_{z} / \pi=0 \mathrm{dd}(\text { even })} S_{o(e)}(q), \\
& {\left[q^{2}-\left(\frac{\omega}{c}\right)^{2}+Q^{2} \sum_{k} H_{(k-q) / 2,(k+q) / 2}^{ \pm}\right] S_{o(e)}(q)} \\
& =Q^{2} \sum_{k} H_{(k-q) / 2,(k+q) / 2}^{ \pm} S_{o(e)}(k)+\frac{2 i \omega}{c L_{z}},
\end{aligned}
$$

and

$$
(k \pm q) L_{z} / 2 \pi=\text { integer; } \quad|k|<2 k_{F}-|q| \text {. }
$$

In the limits $\omega \tau \gg 1$ and $k_{B} \Theta \ll \mu$, Eq. (3.19) for $H_{k_{z_{1} k_{z_{2}}}^{ \pm}}^{ \pm}$can be calculated to

$$
\begin{aligned}
& H_{k_{z_{1}} k_{z_{2}}}^{ \pm}= \frac{1}{2} \omega \tau\left(\frac{1}{i-a^{3}}+\frac{k_{B} \Theta_{D}}{3 \mu a^{3}} \sum_{p=-1}^{1} c_{p} \ln \frac{1-c_{p}}{0-c_{p}}\right) \\
&\left(c_{p}=i a e^{2 \pi i p / 3}\right),
\end{aligned}
$$

where

$$
a^{3}=-\omega \tau\left[1 \pm \omega_{c} / \omega+(\hbar / 2 m \omega)\left(k_{z_{1}}^{2}-k_{z_{2}}^{2}\right)\right] .
$$

The term proportional to the Debye temperature is the electron-phonon contribution and becomes important when

$$
|a| \sim\left(k_{B} \Theta_{D} / \mu\right)^{1 / 2} \ll 1 .
$$

Then Eq. (4.10) can be approximated by

$$
\begin{aligned}
& H_{k_{z_{1}}{ }^{k_{2}}}^{ \pm}=\frac{1}{2} \omega \tau\left[\frac{1}{i-a^{3}}\right.-\frac{\pi k_{B} \Theta_{D}}{3 \mu a^{2}}\left(\operatorname{sgn}(a)+\frac{i}{\sqrt{3}}\right. \\
&\left.\left.+\frac{3 i}{\pi} \sum_{p=1}^{\infty} \frac{(i a)^{3 p-1}}{3 p-1}\right)\right]
\end{aligned}
$$

Equation (4.9) is of nonlocal type and must by the aid of a couple of iterations be solved selfconsistently. To get a finite transmission coef-

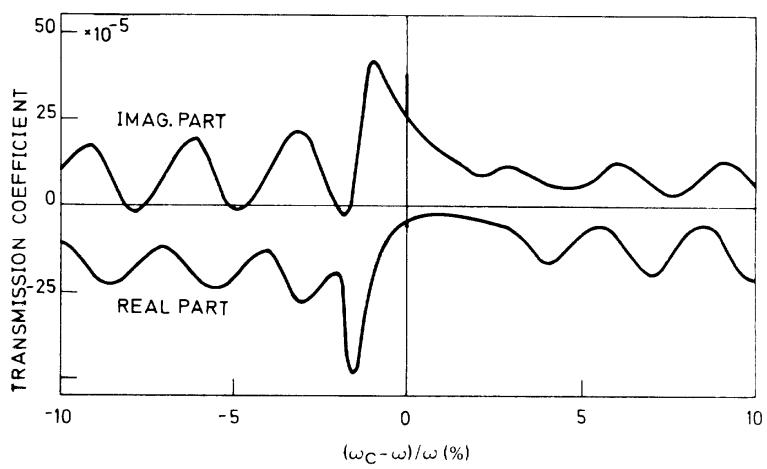

FIG. 1. Transmission coefficient vs the magnetic field for a potassium film with $L_{z}=0.325 \mu \mathrm{m}$ and $\Theta_{D}$ $=91.1 \mathrm{~K}$.

ficient, $q L_{z} / \pi$ has to be discrete, i.e., $L_{z}$ cannot be taken infinite. Two properties of Eq. (4.9) can easily be derived. At first the diagonal term $k=q$ can be subtracted from both sides. In fact, calculations have shown that even the local approximation (zeroth order iteration) of Eq. (4.9) becomes essentially closer to the correct value if the diagonal term is omitted. The second property is the sum rule obtained by summing the Eq. (4.9) with respect to the wave number $q$

$$
\sum_{|q|<2 k_{F}}\left[q^{2}-\left(\frac{\omega}{c}\right)^{2}\right] S_{o(e)}(q)=\frac{4 i}{\pi} \frac{k_{F} \omega}{c} .
$$

The metal is assumed to be very pure, so $\omega \tau$ has been given the large value $300 .^{8}$ The Debye temperature $\Theta_{D}$ is $91.1 \mathrm{~K}$ according to Kittel. ${ }^{12}$ We are interested in finding the structure of the transmission spectrum in the neighborhood of $\omega_{c}=\omega$. Therefore contributions of importance appear only in the minus polarized wave $E_{-}$, because of the magnetic field dependence of Eq. (4.11). Numerical calculations have been performed with both finite and zero Debye temperature, in order to show what influence the electronphonon interaction might have. A general effect when $\Theta_{D}$ is nonzero is that the direct transmitted power $|f|^{2}$ exhibits a very sharp peak at $\omega_{c}=\omega$. For $L_{z}$ less than $0.01 \mu \mathrm{m}$, we do not find any structure of importance apart from this electronphonon peak.

In the Figs. 1 and 2, the transmission coefficient for $L_{z}=0.325$ and $1.00 \mu \mathrm{m}$, respectively, are plotted when $\Theta_{D}$ is nonzero. The necessary number of iterations is decreasing with $\left|\left(\omega_{c}-\omega\right) / \omega\right|$ and varies between five and two. By varying the superposed bias signal $E_{b}$, it has been possible to get two maxima of the total transmitted power $\left|f+E_{b}\right|^{2}$ (Figs. 3 and 4). One peak can mainly be ascribed to the assumption of specular boundary conditions, which yield multiple reflections of the electromagnetic waves. The other much 


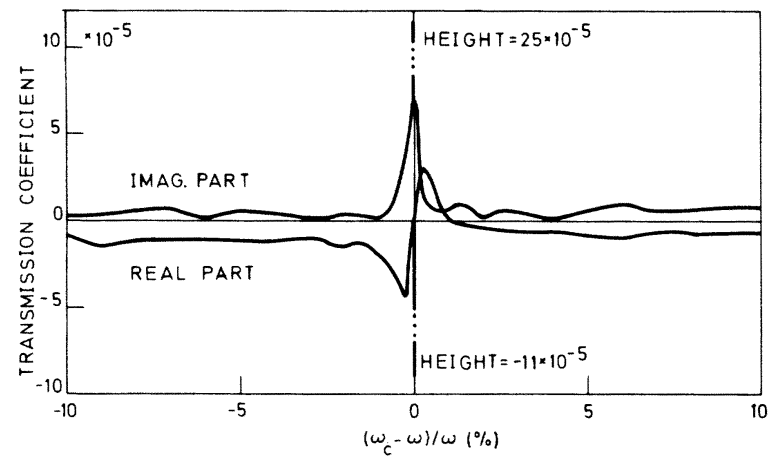

FIG. 2. Transmission coefficient vs the magnetic field for a potassium film with $L_{*}=1.00 \mu \mathrm{m}$ and $\Theta_{D}$ $=91.1 \mathrm{~K}$. The electron-phonon peak height is indicated.

sharper peak is entirely connected with the electron-phonon interaction. The relative width of the electron-phonon peak can be obtained from Eqs. (4.11) and (4.12) as

$$
\frac{\partial\left(\omega_{c}-\omega\right)}{\omega} \approx\left(\frac{k_{B} \Theta_{D}}{\mu}\right)^{3 / 2} \frac{1}{\omega \tau} .
$$

The numerical value is not larger than $10^{-4} \%$ in this case with the very clean potassium film. Therefore, according to Eq. (4.15) the peak might be observable if the metal is so dirty that $\omega \tau$ $\leqslant 0.1$. This is a very rough estimation because the important limitation $\omega \tau \gg 1$ of the derived expressions in Secs. III and IV has now been ignored.

The difference between the transmission coeffor nonzero and zero Debye temperature is plotted in the Figs. 5 and 6 for $L_{z}=0.325$ and $1.00 \mu \mathrm{m}$, respectively. As can be seen, the relative influence of the electron-phonon interaction becomes more important at $\omega_{c} \approx \omega$ when the film thickness increases.

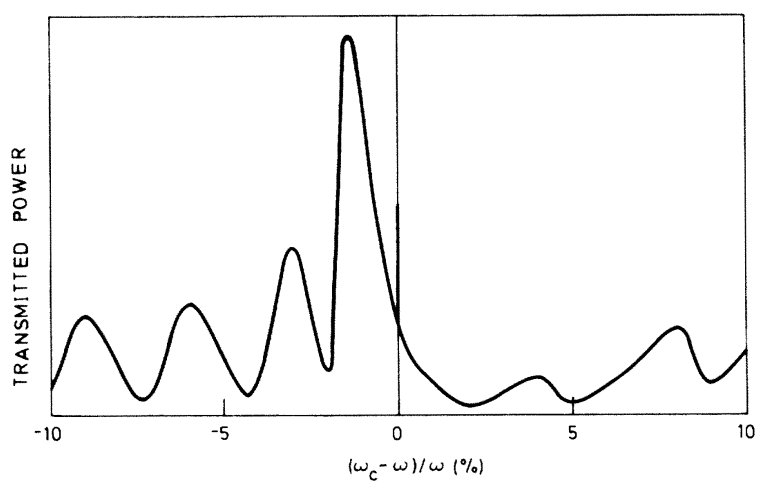

FIG. 3. Transmitted power vs the magnetic field for $L_{\mathrm{z}}=0.325 \mu \mathrm{m}$ and $\Theta_{D}=91.1 \mathrm{~K}$. The added bias signal is $1.5 \times 10^{-5} e^{1.26 i}$ times the incoming signal.

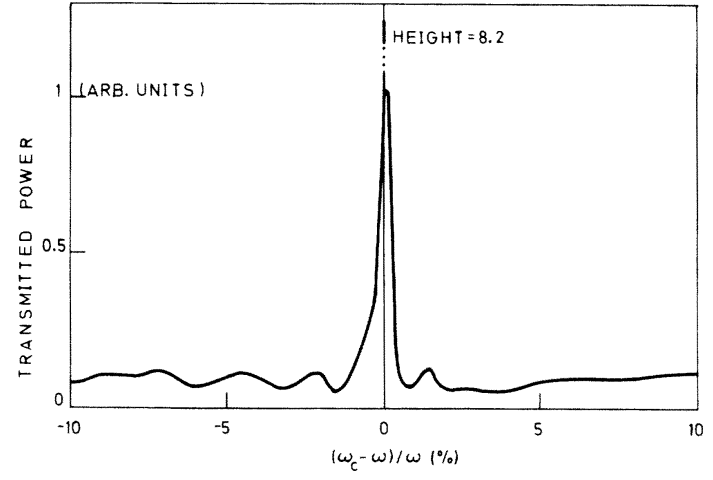

FIG. 4. Transmitted power vs the magnetic field for $L_{\mathrm{s}}=1.00 \mu \mathrm{m}$ and $\Theta_{D}=91.1 \mathrm{~K}$. The added bias signal is $5.05 \times 10^{-5} e^{1.83 i}$ times the incoming signal.

\section{CONCLUSIONS}

The direct transmitted signal across a metal film (the transmission coefficient) varies linearly with the magnetic field, if the film thickness is less than $0.01 \mu \mathrm{m}$ and the electron-phonon interaction is zero. The transmission coefficient begins to vary periodically with the magnetic field when the magnitude of $L_{z}$ becomes larger than about $0.1 \mu \mathrm{m}$. The largest amplitude appears in the vicinity of $\omega_{c}=\omega$. At finite electron-phonon interaction the transmission coefficient is changed. The deviation is especially important when $\omega_{c}$ is very close to $\omega$, where a sharp peak appears. The width of this peak decreases with increasing $\omega \tau$. A rough estimation shows that $\omega \tau$ must not be greater than about 0.1 , if the peak shall be observable. Then, however, the film thickness must be small in order to transmit any electromagnetic power. In the vicinity of the peak the influence of the electron-phonon interaction is not negligible

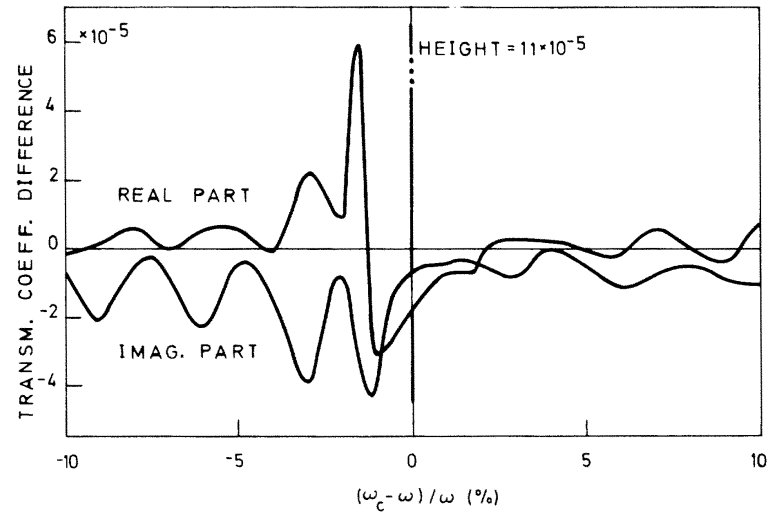

FIG. 5. Difference between the transmission coefficients in the cases $\Theta_{D}=91.1 \mathrm{~K}$ and $\Theta_{D}=0.0 \mathrm{~K} . L_{\text {s }}$ $=0.325 \mu \mathrm{m}$. 


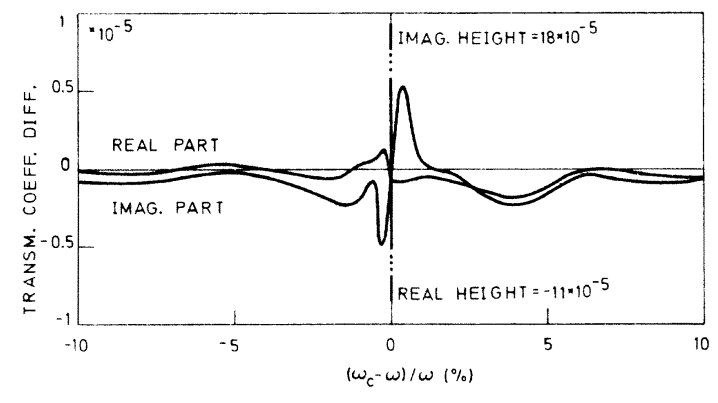

FIG.6. Difference between the transmission coefficients in the cases $\Theta_{D}=91.1 \mathrm{~K}$ and $\Theta_{D}=0.0 \mathrm{~K}$. $L_{*}$ $=1.00 \mu \mathrm{m}$.

even for large $\omega \tau$.

As the computer time increases proportional to the second power of the film thickness, there must be a limiting point for the calculations. Unfortunately, this occurred to us when $L_{z}$ was of the magnitude $1 \mu \mathrm{m}$. However, as can be seen in
Fig. 2, rather rapid variations are present in the transmission coefficient near $\omega_{c}=\omega$ even for so thin films. By adding a signal, which is the sum of an unknown leakage signal around the metal and the variable exterior bias signal, it therefore also becomes possible to obtain peak structure in the total transmitted microwave power (Fig. 4).

By comparing the result for the different film thicknesses, we suggest that by the aid of a very rapid computer it would be possible to get as sharp transmission peaks as has been obtained experimentally by Philips et al. ${ }^{8}$ with a potassium film of the thickness $32.5 \mu \mathrm{m}$. The only statement we can make regarding the positions of these peaks are that they must be close to $\omega_{c}=\omega$.

\section{ACKNOWLEDGMENTS}

The author wants to thank K. Saermark for his hospitality at Physics Lab. I of the Technical University of Denmark. He is also grateful to N. E. Christensen, J. Mygind, and M. R. Samuelsen for many valuable discussions.
${ }^{1}$ P. R. Antoniewitz, Phys. Rev. 185, 863 (1969).

${ }^{2}$ J. F. Carolan and A. P. van Gelder, Phys. Rev. Lett。 25, 1433 (1970).

${ }^{3}$ A. P. van Gelder, Phys. Rev. 187, 833 (1969).

${ }^{4}$ P. M. Platzman and K. C. Jacobs, Phys. Rev. 134, A974 (1964).

${ }^{5}$ T. M. Rice, Ann。 Phys. (N.Y.) 31, 100 (1965).

${ }^{6}$ R. E. Prange and L. P. Kadanoff, Phys. Rev. 134, A566 (1964).

${ }^{7}$ D. R. Fredkin and R. Freedman, Phys. Rev。B $\underline{9}, 360$
(1974).

${ }^{8}$ T. G. Phillips, G. A. Baraff, and G. L. Dunifer, Phys. Rev. Lett. 30, 274 (1973)。

${ }^{9}$ G. A。 Baraff, Phys。 Rev. B 9 , 4008 (1974).

${ }^{10} \mathrm{H}$. Scher and T. Holstein, Phys. Rev. 148, 598 (1966).

${ }^{11}$ L. V. Keldysh, Zh. Eksp. Teor. Fiz. $\underline{47}, 1515$ (1964) [Sov. Phys. -JETP 20, 1018 (1965)].

${ }^{12} \mathrm{C}$. Kittel, Introduction to Solid State Physics, 3rd ed. (Wiley, New York, 1966). 